\title{
Complications and 3-month outcomes of children with hydrocephalus treated with ventriculoperitoneal shunts in Malawi
}

\author{
Trista Reid, MD, MPH, ${ }^{1}$ Joanna Grudziak, MD, MPH, ${ }^{1}$ Nidia Rodriguez-Ormaza, MD, MPH, ${ }^{2}$ \\ Rebecca G. Maine, MD, MPH, ${ }^{1}$ Nelson Msiska, ${ }^{3}$ Carolyn Quinsey, MD, ${ }^{4}$ and \\ Anthony Charles, MD, MPH'1,3
}

\begin{abstract}
Departments of ${ }^{1}$ Surgery, ${ }^{2}$ Epidemiology, and ${ }^{4}$ Neurosurgery, University of North Carolina at Chapel Hill, North Carolina; and ${ }^{3}$ Department of Surgery, Kamuzu Central Hospital, Lilongwe, Malawi
\end{abstract}

\begin{abstract}
OBJECTIVE Hydrocephalus is the most common pediatric neurosurgical condition, with a high prevalence in low- and middle-income countries. Untreated, hydrocephalus leads to neurological disability or death. The epidemiology and outcomes of hydrocephalus treated by ventriculoperitoneal (VP) shunts in Sub-Saharan Africa are not well defined and vary by region. The aim of the present study was to examine the mortality and morbidity rates and predictors of mortality in children treated by VP shunt placement for hydrocephalus at Kamuzu Central Hospital in Lilongwe, Malawi.

METHODS This is a prospective study of 100 consecutive children presenting with hydrocephalus who were treated with VP shunt placement from January 2015 to August 2017. Demographics, nutritional status, maternal characteristics, developmental delay, shunt complications, readmissions, and in-hospital and 3-month mortality data were collected. Multivariate logistic regression was used to identify predictors of death within 3 months of surgery.

RESULTS Overall, $46 \%$ of participants were female, with an average age of $5.4 \pm 3.7$ months at the time of surgery. The majority of patients were term deliveries (87.8\%) and were not malnourished (72.9\%). Only $10.8 \%$ of children were diagnosed with meningitis before admission. In-hospital and 3-month mortality rates were $5.5 \%$ and $32.1 \%$, respectively. The only significant association with mortality was maternal age, with older maternal age demonstrating decreased odds of 3-month mortality (OR 0.9, 95\% $\mathrm{Cl} 0.8-1.0, \mathrm{p}=0.045)$.

CONCLUSIONS Surgical management of hydrocephalus with VP shunts portends a high mortality rate in Malawi. The association of younger maternal age with mortality is likely a proxy for social determinants, which appear to contribute as much to mortality as patient factors. VP shunting is inadequate as a sole surgical management of hydrocephalus in resource-limited settings.
\end{abstract}

https://thejns.org/doi/abs/10.3171/2019.2.PEDS18325

KEYWORDS hydrocephalus; ventriculoperitoneal shunt; pediatrics; global surgery

$\mathrm{H}$ YDROCEPHALUS is the most common neurosurgical condition in children; the congenital form affects $0.5-1$ per 1000 live births, and the neonatal acquired form affects $3-5$ per 1000 live births..$^{15}$ It is also the most common surgically correctable clinical condition in the pediatric population worldwide. ${ }^{8}$ The prevalence of hydrocephalus is greatest in low- and middle-income countries (LMICs), where access to neurosurgical care is limited. This predisposition is attributed to higher birth rates, poor prenatal care, and a higher incidence of meningitis in the perinatal and neonatal period. While specific epidemiology of hydrocephalus is not available for every country in Sub-Saharan Africa, the annual incidence of hydrocephalus in Sub-Saharan Africa is approximately 145 per 100,000 births, which is almost twice the rate found in industrialized nations. ${ }^{2,5,19}$ Hydrocephalus accounts for $32 \%$ of congenital neurosurgical conditions in Nigeria and 59\% of all neurosurgical conditions in Uganda. ${ }^{15}$

ABBREVIATIONS CPC = choroid plexus cauterization; ETV = endoscopic third ventriculostomy; $\mathrm{HIC}=$ high-income country; $\mathrm{KCH}=\mathrm{Kamuzu}$ Central Hospital; LMICs = low- and middle-income countries; ROC = receiver operating characteristic; VP = ventriculoperitoneal.

SUBMITTED May 31, 2018. ACCEPTED February 28, 2019.

INCLUDE WHEN CITING Published online May 10, 2019; DOI: 10.3171/2019.2.PEDS18325. 
Most cases of pediatric hydrocephalus in Sub-Saharan Africa are classified as obstructive, and common causes include spina bifida and postinfectious acquired hydrocephalus. In contrast, the most common etiologies in highincome countries (HICs) are congenital and prematurityrelated intraventricular hemorrhage. ${ }^{15}$ The treatment rate of pediatric hydrocephalus in Sub-Saharan Africa was recently estimated to be less than $10 \% .{ }^{19}$ Untreated hydrocephalus can result in permanent neurological disability and death. ${ }^{13,22}$ In a 1962 study of the natural history of untreated hydrocephalus based on 182 cases, the chances that a hydrocephalic child diagnosed soon after birth would survive to adulthood were estimated to be $20 \%-23 \%$, rising to $26 \%$ for children evaluated at 3 months, and $50 \%$ for children who survived to at least 1 to 2 years of age. The overall mortality rate during the 20 years of the study was $49 \%$. Only $32 \%$ of surviving children displayed full physical functioning, with $48 \%$ showing severe disability. ${ }^{13}$ Even with treatment, outcomes in the developing world can be unsatisfactory, with one study reporting that $60 \%$ of patients had poor outcomes when rated based on visual, motor, and seizure criteria. ${ }^{10}$

Treatment of hydrocephalus in LMICs is limited by lack of resources, neurosurgical personnel, and infrastructure. ${ }^{15}$ The current standard of pediatric hydrocephalus care in Malawi, as in other nations, is ventriculoperitoneal (VP) shunt placement. VP shunts are prone to complications and require close monitoring. VP shunt obstruction, migration, and infection are common complications that require revision or replacement. Because of this, children with VP shunts require more resources for both acute and long-term management than do children with other conditions managed in hospitals. ${ }^{15}$ Even in HICs, where VP shunt procedures represent a very small percentage of overall pediatric procedures performed, they account for the second-highest rate of hospital admissions in pediatric patients. ${ }^{9} \mathrm{VP}$ shunt failure reaches $50 \%$ at 2 years in HICs, with an infection rate of $4.1 \%-20.5 \% .{ }^{6,20}$ In spite of the complications of VP shunt placement, studies in HICs show that it represents an economically and socially effective intervention for this morbid condition..$^{15,18}$ Given the resource constraints and difficulties faced by Sub-Saharan African families seeking care for initial and subsequent hydrocephalus complications, the mortality and morbidity rates associated with pediatric hydrocephalus in this setting are likely much higher than in the developing world. ${ }^{4,15}$

Currently, very limited data are available regarding the long-term outcomes of Sub-Saharan African children following VP shunt placement. Given the paucity of data in this region, we sought to characterize outcomes, as well as patient and social characteristics leading to poor outcomes in a resource-limited setting. The aim of our study was to examine the mortality and morbidity rates and predictors of mortality in children treated for hydrocephalus with VP shunt placement at the Kamuzu Central Hospital (KCH) in Lilongwe, Malawi.

\section{Methods}

This is a prospective study of 100 children (less than 18 years of age) presenting consecutively with hydrocepha- lus who were treated with VP shunt placement at $\mathrm{KCH}$ in Lilongwe, Malawi, from January 2015 to August 2017. $\mathrm{KCH}$, one of four tertiary referral centers in Malawi, has an approximate catchment area of 6 million people, 900 beds, and two clinical officers who perform VP shunt placements, with 5 and 8 years of shunt surgery experience. A neurosurgeon was available at $\mathrm{KCH}$ during the study period. A pediatric step-down unit is available, but there is no pediatric intensive care unit. Available imaging modalities include head ultrasound and, intermittently, $\mathrm{CT}$; hydrocephalus patients do not currently undergo CT or MRI evaluation.

The primary outcomes of interest were postoperative mortality following VP shunt surgery, specifically in-hospital and 3-month mortality rates after surgery. Secondary outcomes included 6-month mortality, hospital readmission, shunt infection, shunt malfunction/malposition, excessive CSF drainage, child growth, and the presence of developmental delay or disability. As almost $50 \%$ of patients lacked mortality data at the 6-month point, these data were not used as a primary outcome.

The secondary outcomes were determined by the clinical officers in the hospital or at the time of follow-up via home visits. Shunt infection was defined by the Infectious Society of America's practice guidelines, including erythema or tenderness over the shunt; new-onset headache, nausea, lethargy, or change in mental status in the appropriate clinical context; bacteremia without other identified source; or signs or symptoms of peritonitis in the absence of another cause..$^{23}$ Exposed shunts were considered suspected infections and all underwent revision. Child growth was determined by adherence to the child's growth curve based on the WHO growth charts for head circumference, length, and weight. Developmental delay was defined as the child not meeting one of the Centers for Disease Control and Prevention developmental milestones for his or her age group. ${ }^{3}$

Variables collected include baseline demographic and clinical characteristics of the children, such as age at time of VP shunt insertion, sex, weight, maternal and paternal age and medical history, nutritional status, prematurity (delivered earlier than 37 weeks' gestation), mode of delivery, reason for presentation, head circumference, and opening pressure, as well as secondary outcomes. The determination of nutritional status was based on birth weight for age or clinical findings of temporal wasting or scaphoid abdomen. Head circumference measures depend on a number of variables including age and sex, thus standard WHO z-scores for head circumference were used. Opening pressure was defined as normal, moderate, or high based on the clinical judgment of the clinical officers inserting the shunts. Prior treatment of meningitis was documented as well, but the etiology of hydrocephalus was difficult to assess in this resource-poor setting and any history of recent febrile illness was confounded by a high incidence of malarial and diarrheal disease. We used the median age of 25 year to categorize maternal age as older and younger. Birth weight was categorized using the WHO's birth weight standard classification.

All data, including follow-up, were collected by a senior data clerk. Follow-up visits and examinations were 


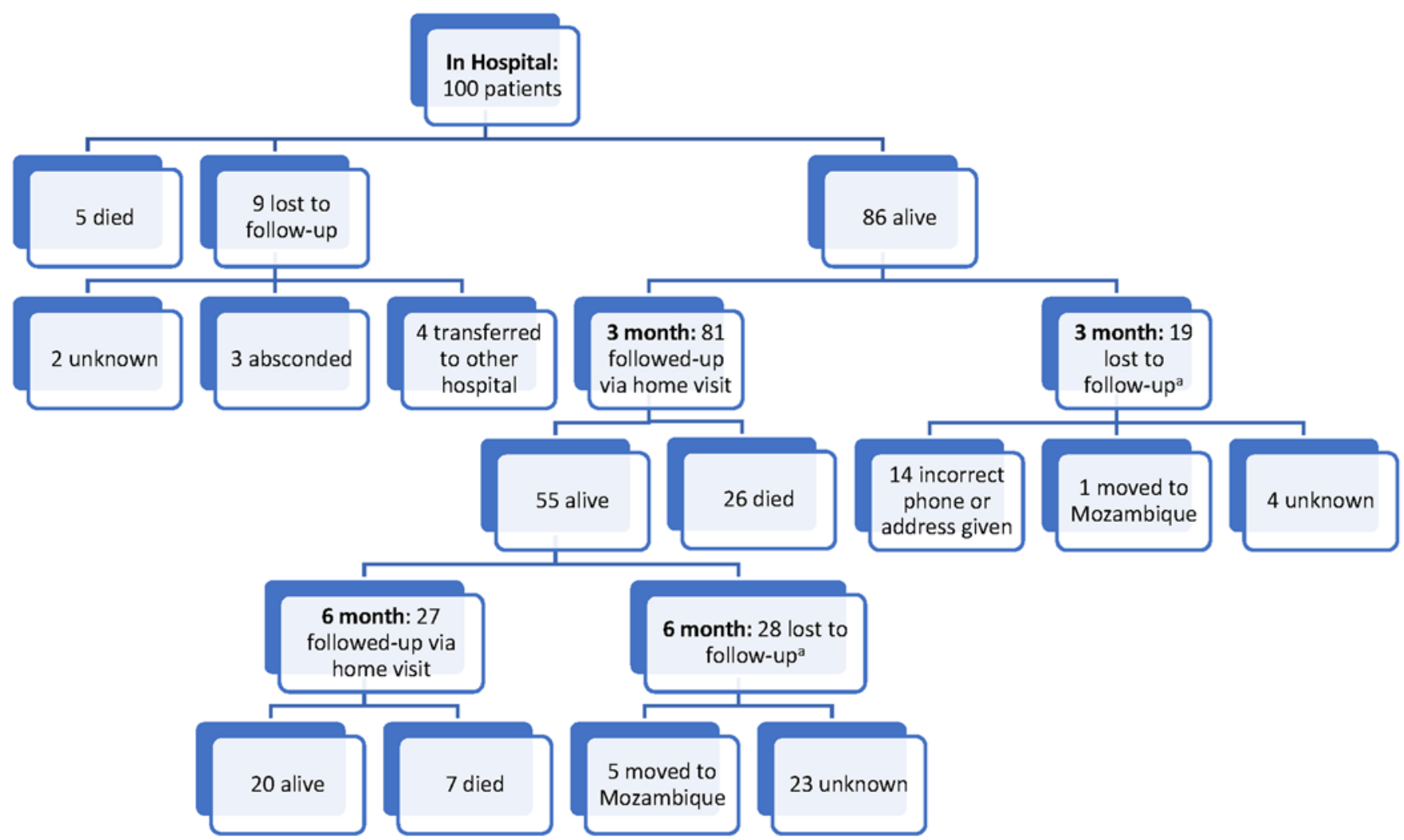

FIG. 1. Patient follow-up over 6 months. aPatients were lost to follow-up despite attempts to reach them by cell phone, home visit, and asking the village elder. Figure is available in color online only.

conducted by a senior data clerk by home visit at the last known address of residence for the child. The clerk initially called families via the cell phone number on file. If this method was not successful, the clerk would visit the last known address. If the family no longer was present at the address, the clerk would contact the village elders to determine a new address. Follow-up data were available for $91 \%$ of patients during hospitalization; $81 \%$ of patients at 3 months; and 53\% of patients at 6 months (Fig. 1). Follow-up in a resource-poor setting is difficult and major barriers in Malawi included movement of families to Mozambique, poor reporting by family members, and the degree of investment and documentation performed by the clerks. These factors account for variable followup at 6 months and even during hospitalization. Additionally, families absconding from the hospital or transferring hospitals affected completeness of in-hospital data. Cause of death proved incredibly difficult to assess given family reporting and budgetary constraints, to the point that these data were not meaningful.

Sample size was affected by numerous factors, including budgetary and time constraints; however, 100 patients were determined to be an adequate representative population based on similar sample sizes among other studies in resource-poor areas., ${ }^{4,10,16,17}$ Bivariate analysis compared patient and parental characteristics by mortality status at 3 months. We also present patients' adverse outcomes in-hospital and at 3 months postsurgery. We used the chisquare and Fisher's exact tests to compare categorical variables, and the Kruskal-Wallis test to compare medians of nonnormally distributed continuous variables (patient's age at surgery, father's age, and head circumference zscore.) The Kruskal-Wallis tests were found to be equivalent to the Mann-Whitney U-test.

We used multivariable logistic regression to calculate odds ratios and $95 \%$ confidence intervals for the association between 3 potential predictors of 3-month mortality determined a priori based on knowledge of risk factors and consideration of bivariate analysis: nutritional status, age in months at time of surgery, and mother's age. Furthermore, we calculated the area under the receiver operating characteristic (ROC) curve, to determine if our model adequately predicted mortality at 3 months. We used the Hosmer-Lemeshow goodness-of-fit test to assess our model fit. A p value of less than 0.05 was used to determine statistical significance. Analyses were conducted using SAS statistical software (version 9.4; SAS Institute Inc.).

This protocol was approved by both the Malawian National Health Services Research Council and the University of North Carolina at Chapel Hill Institutional Review Board. Informed consent for this data collection was obtained from families in their primary language, and all were free to withdraw from the study at any point.

\section{Results}

Of the 100 patients undergoing VP shunt placement, $46(46.0 \%)$ were female. The median age at the time of surgery was 4.5 months (IQR 3.0-7.0 months). Fifty-nine (74.7\%) of 79 participants had a birth weight at or above 
TABLE 1. Patient and parental characteristics total and by mortality status

\begin{tabular}{|c|c|c|c|c|}
\hline \multirow[b]{2}{*}{ Variable } & \multicolumn{3}{|c|}{ No. of Patients (\%) or Median (IQR) } & \multirow[b]{2}{*}{ p Value } \\
\hline & Total $(n=100)$ & Dead by 3 Mos $(n=26)$ & Alive by 3 Mos $(n=55)$ & \\
\hline Age (mos) & $4.5(3.0-7.0)$ & $4(3.0-6.0)$ & $5(3.0-7.0)$ & 0.666 \\
\hline Sex & & & & 0.675 \\
\hline Female & $46 / 100(46.0 \%)$ & $11 / 26(42.3 \%)$ & $26 / 55(47.3 \%)$ & \\
\hline Male & $54 / 100(54.0 \%)$ & $15 / 26(57.7 \%)$ & $29 / 55(52.7 \%)$ & \\
\hline Mother's age (yrs) & $25(21-29)$ & $22.5(20-26)$ & $25(22-29)$ & 0.027 \\
\hline$<25$ & $47 / 99(47.5 \%)$ & $16 / 26(61.5 \%)$ & $23 / 54(42.6 \%)$ & 0.112 \\
\hline$\geq 25$ & $52 / 99(52.5 \%)$ & $10 / 26(38.5 \%)$ & $31 / 54(57.4 \%)$ & \\
\hline Father's age (yrs) & $30(25-35)$ & $26(24-32)$ & $30(26-34)$ & 0.251 \\
\hline Mother's HIV status & & & & 0.169 \\
\hline Positive & $7 / 93(7.5 \%)$ & $1 / 25(4.0 \%)$ & $6 / 53(11.3 \%)$ & \\
\hline Negative & $86 / 93(92.5 \%)$ & $20 / 25(80.0 \%)$ & $47 / 53(88.7 \%)$ & \\
\hline Folic acid prepregnancy & & & & 0.745 \\
\hline Yes & $14 / 98(14.3 \%)$ & $5 / 25(20.0 \%)$ & $8 / 54(14.8 \%)$ & \\
\hline No & $84 / 98(85.7 \%)$ & $20 / 25(80.0 \%)$ & $46 / 54(85.2 \%)$ & \\
\hline Teratogenic drug use: alcohol & & & & 1.000 \\
\hline Yes & $1 / 100(1.0 \%)$ & $0 / 24(0.0 \%)$ & $1 / 53(1.9 \%)$ & \\
\hline No & $99 / 100(99.0 \%)$ & $24 / 24(100.0 \%)$ & $52 / 53(98.1 \%)$ & \\
\hline Delivery mode & & & & 0.411 \\
\hline Vaginal delivery & $71 / 96(74.0 \%)$ & $22 / 26(84.6 \%)$ & $36 / 52(69.2 \%)$ & \\
\hline Cesarean section & $14 / 96(14.6 \%)$ & $2 / 26(7.7 \%)$ & 9/52 (17.3\%) & \\
\hline Assisted vaginal delivery & $11 / 96(11.5 \%)$ & $2 / 26(7.7 \%)$ & $7 / 52(13.5 \%)$ & \\
\hline Gestational age (wks) & & & & 0.677 \\
\hline Preterm $(<37)$ & $12 / 98(12.3 \%)$ & $3 / 26(11.5 \%)$ & $4 / 54(7.4 \%)$ & \\
\hline Term & $86 / 98(87.8 \%)$ & $23 / 26(88.5 \%)$ & $50 / 54(92.6 \%)$ & \\
\hline Birth weight $(\mathrm{kg})$ & & & & 1.000 \\
\hline$<2.5$ & $20 / 79(25.3 \%)$ & $4 / 21(19.1 \%)$ & $9 / 41(22.0 \%)$ & \\
\hline$\geq 2.5$ & $59 / 79(74.7 \%)$ & $17 / 21(81.0 \%)$ & $32 / 41(78.0 \%)$ & \\
\hline Head circumference (z-score) & $9(5.9-12.0)$ & $7.1(3.8-10.6)$ & $9.9(6.2-13.1)$ & 0.031 \\
\hline Nutritional status & & & & 0.199 \\
\hline Nourished & $70 / 96(72.9 \%)$ & $8 / 25(32.0 \%)$ & $10 / 53(18.9 \%)$ & \\
\hline Malnourished & $26 / 96(27.1 \%)$ & $17 / 25(68.0 \%)$ & $43 / 53(81.1 \%)$ & \\
\hline \multicolumn{5}{|l|}{ History of treatment for meningitis } \\
\hline Yes & $10 / 93(10.8 \%)$ & $2 / 22(9.1 \%)$ & $6 / 51(11.8 \%)$ & 0.168 \\
\hline No & $83 / 93(89.2 \%)$ & $20 / 22(90.9 \%)$ & $45 / 51(88.2 \%)$ & \\
\hline \multicolumn{5}{|l|}{ Reasons for presentation at hospital } \\
\hline Large head & $94 / 99(95.0 \%)$ & $24 / 26(92.3 \%)$ & $52 / 54(96.3 \%)$ & 0.592 \\
\hline Convulsions & $22 / 99(22.2 \%)$ & $5 / 26(19.2 \%)$ & $13 / 54(24.1 \%)$ & 0.778 \\
\hline Delayed milestones & $5 / 98(5.1 \%)$ & $3 / 26(11.5 \%)$ & $2 / 54(3.7 \%)$ & 0.323 \\
\hline Vomiting & $13 / 99(13.1 \%)$ & $4 / 26(15.4 \%)$ & $6 / 54(11.1 \%)$ & 0.720 \\
\hline Irritability & $26 / 99(26.3 \%)$ & $3 / 26(11.5 \%)$ & $20 / 54(37.0 \%)$ & 0.020 \\
\hline Infection & $24 / 99(24.2 \%)$ & $4 / 26(15.4 \%)$ & $17 / 54(31.5 \%)$ & 0.177 \\
\hline Other reasons & $7 / 99(7.1 \%)$ & $1 / 25(4.0 \%)$ & $5 / 55(9.1 \%)$ & 0.660 \\
\hline Ultrasound scan & & & & 0.601 \\
\hline Hydrocephalus & $91 / 91(100.0 \%)$ & $25 / 25(100.0 \%)$ & $53 / 53(100.0 \%)$ & \\
\hline Additional abnormal findings* & $8 / 91(8.8 \%)$ & $2 / 25(8.0 \%)$ & $6 / 53(11.3 \%)$ & \\
\hline Opening pressure $\left(\mathrm{cm} \mathrm{H}_{2} \mathrm{O}\right)$ & & & & 0.833 \\
\hline Low/normal & $1 / 71(1.4 \%)$ & $0 / 16(0.0 \%)$ & $1 / 42(2.4 \%)$ & \\
\hline Moderate & $36 / 74(48.6 \%)$ & $9 / 16(56.3 \%)$ & $19 / 41(46.3 \%)$ & \\
\hline High & $37 / 74(50.0 \%)$ & $7 / 16(43.8 \%)$ & $21 / 41(51.2 \%)$ & \\
\hline
\end{tabular}

* Additional abnormal findings on ultrasound included hemorrhage, tumor, midline shift, and other infrequent findings. 
$2.5 \mathrm{~kg}$, and $86(87.8 \%)$ of 98 were term births, with 82 $(85.4 \%)$ of 96 undergoing vaginal or vaginal assisted deliveries. Only $10(10.8 \%)$ of 93 patients were ever treated for meningitis prior to hydrocephalus assessment. Malnutrition was present in 26 (27.1\%) of 96 patients at the time of surgery, with a mean weight at surgery of $6.3 \mathrm{~kg}( \pm 2.5$ $\mathrm{kg}$ ) (Table 1). The median maternal age was 25 years (IQR 21-29 years), and the median paternal age was 30 years (IQR 25-35 years). Seven (7.5\%) of 93 mothers were HIV positive, $7(7.5 \%)$ of 93 had an unknown HIV status, while $86(92.5 \%)$ of 93 were HIV negative. Only $14(14.3 \%)$ of 98 mothers reported folic acid supplementation during pregnancy. One (1\%) of 100 mothers admitted to alcohol use during pregnancy (Table 1).

An ultrasound report was available for 91 of the children; no child underwent CT scanning or MRI. Of those children with an ultrasound report, hemorrhage was identified in $1(1.1 \%)$ of 91 patients, midline shift in $7(7.7 \%)$ of 91 patients, and $66(72.5 \%)$ of 91 patients had communicating hydrocephalus. Imaging did not identify any tumors. The opening pressure was moderate for 36 (48.6\%) of 74 patients and high for $37(50.0 \%)$ of 74 patients. The most common reasons for presentation included large head in $94(95.0 \%)$ of 99 patients, irritability in $26(26.3 \%)$ of 99 patients, and convulsions in $22(22.2 \%)$ of 99 patients (Table 1).

Five $(5.5 \%)$ of 91 patients died while in the hospital. Of the 81 patients with 3-month postoperative follow-up, 26 (32.1\%) had died. Of the 53 patients with 6-month postoperative follow-up, $33(62.3 \%)$ had died. Table 2 shows overall and 3-month complications by mortality status. None of the complications at 3 months were significantly associ-

TABLE 2. Complications at hospitalization and at the 3-month follow-up

\begin{tabular}{|c|c|c|c|c|}
\hline \multirow[b]{2}{*}{ Variable } & \multicolumn{3}{|c|}{ No. of Patients (\%) } & \multirow[b]{2}{*}{ p Value } \\
\hline & Total $(n=100)$ & Dead by 3 Mos $(n=26)$ & Alive by 3 Mos $(n=55)$ & \\
\hline Shunt revision & & & & 1.000 \\
\hline Yes & $5 / 88(5.7 \%)$ & $1 / 22(4.6 \%)$ & $3 / 51(5.9 \%)$ & \\
\hline No & $82 / 88(93.2 \%)$ & $21 / 22(95.5 \%)$ & $48 / 51(94.1 \%)$ & \\
\hline Cause of shunt revision & & & & 0.250 \\
\hline Blocked shunt & $1 / 5(20.0 \%)$ & $1 / 2(50.0 \%)$ & $0 / 3(0.0 \%)$ & \\
\hline Exposed shunt & $4 / 5(80.0 \%)$ & $1 / 2(50.0 \%)$ & $3 / 3(100.0 \%)$ & \\
\hline Reason for longer stay & & & & 0.300 \\
\hline Bloody CSF & $1 / 11(9.1 \%)$ & $1 / 3(33.3 \%)$ & $0 / 7(0.0 \%)$ & \\
\hline Hospital operation time & $1 / 11(9.1 \%)$ & $0 / 3(0.0 \%)$ & $1 / 7(14.3 \%)$ & \\
\hline Opening space & $1 / 11(9.1 \%)$ & $0 / 3(0.0 \%)$ & $1 / 7(14.3 \%)$ & \\
\hline Operating space & $1 / 11(9.1 \%)$ & $1 / 3(33.3 \%)$ & $0 / 7(0.0 \%)$ & \\
\hline Operating time & $2 / 11(18.2 \%)$ & $1 / 3(33.3 \%)$ & $1 / 7(14.3 \%)$ & \\
\hline Other infections & $1 / 11(9.1 \%)$ & $0 / 3(0.0 \%)$ & $0 / 7(0.0 \%)$ & \\
\hline Pneumonia & $1 / 11(9.1 \%)$ & $0 / 3(0.0 \%)$ & $1 / 7(14.3 \%)$ & \\
\hline Shortage of shunt & $3 / 11(27.3 \%)$ & $0 / 3(0.0 \%)$ & $3 / 7(42.9 \%)$ & \\
\hline Overdrainage & & & & 1.000 \\
\hline Yes & $1 / 79(1.3 \%)$ & $0 / 19(0.0 \%)$ & $1 / 47(2.1 \%)$ & \\
\hline No & $78 / 79(98.7 \%)$ & $19 / 19(100.0 \%)$ & $46 / 47(97.9 \%)$ & \\
\hline Meningitis & & & & 1.000 \\
\hline Yes & $3 / 79(3.8 \%)$ & $0 / 19(0.0 \%)$ & $2 / 47(4.3 \%)$ & \\
\hline No & $76 / 79(96.2 \%)$ & $19 / 19(100.0 \%)$ & $45 / 47(95.7 \%)$ & \\
\hline Other complications & & & & 1.000 \\
\hline Febrile illness & $3 / 100(3.0 \%)$ & $1 / 26(3.8 \%)$ & $1 / 55(1.8 \%)$ & \\
\hline Umbilical hernia & $1 / 100(1.0 \%)$ & $0 / 26(0.0 \%)$ & $1 / 55(1.8 \%)$ & \\
\hline Burst abdomen & $1 / 100(1.0 \%)$ & $0 / 26(0.0 \%)$ & $1 / 55(1.8 \%)$ & \\
\hline VP shunt exposure & & & & 0.565 \\
\hline Yes & $4 / 54(7.4 \%)$ & $1 / 13(7.7 \%)$ & $3 / 33(9.1 \%)$ & \\
\hline No & $50 / 54(92.6 \%)$ & $12 / 13(92.3 \%)$ & $30 / 33(90.9 \%)$ & \\
\hline Meeting milestones & & & & 1.000 \\
\hline Yes & $19 / 54(35.2 \%)$ & 4/13 (30.8\%) & $12 / 33(36.4 \%)$ & \\
\hline No & $35 / 54(64.8 \%)$ & $9 / 13(69.2 \%)$ & $21 / 33(63.6 \%)$ & \\
\hline Eating better & & & & 1.000 \\
\hline Yes & $34 / 54(63.0 \%)$ & $9 / 13(69.2 \%)$ & $21 / 33(63.6 \%)$ & \\
\hline No & $20 / 54(37.0 \%)$ & $4 / 13(30.8 \%)$ & $12 / 33(36.4 \%)$ & \\
\hline
\end{tabular}


TABLE 3. Multivariable analysis of predictors of all-cause mortality by 3 months after surgery

\begin{tabular}{ccc}
\hline Factor & OR $(95 \% \mathrm{Cl})$ & p Value \\
\hline Nutritional status & & \\
\hline Malnourished & $2.0(0.6-6.3)$ & 0.256 \\
\hline Nourished & Reference & \\
\hline Age (mos) & $1.1(0.9-1.2)$ & 0.364 \\
\hline Mother's age (yrs) & $0.9(0.8-1.0)$ & 0.045 \\
\hline
\end{tabular}

Boldface type indicates statistical significance.

ated with mortality. In-hospital complications included one patient with overdrainage of CSF, one patient with meningitis, and one patient with an exposed shunt the same day of placement who underwent immediate revision. Of the 4 $(8.7 \%)$ of 46 patients who had documentation of an exposed shunt at 3 months, all underwent revision and $1(1.9 \%)$ had died by 3 months. In total, 5 (5.7\%) of 88 patients underwent shunt revisions, primarily for exposed shunts.

Three (3.8\%) of 79 patients developed meningitis: 1 in-hospital patient who was lost to follow-up at 3 months and 2 patients at the 3-month visit. There were no reported wound infections at the 3-month follow-up visit, but the 3 exposed shunts were considered suspected infections, and all patients underwent revisions. At the 3-month follow-up visit, only $19(35.2 \%)$ of 54 children were meeting developmental milestones.

Table 3 shows the multivariable analysis of potential predictors of all-cause mortality by 3 months after surgery. Only older maternal age was significantly associated with decreased odds of 3-month mortality (OR 0.9, 95\% CI 0.8$1.0, \mathrm{p}=0.045)$. This association persisted when excluding patients with mothers $\leq 16$ years of age but not $\leq 18$ years. Nonsignificant associations were found for a patient's age at surgery (OR 1.1, 95\% CI 0.9-1.2, p = 0.364) and nutritional status (OR 2.0,95\% CI 0.6-6.3, p = 0.256) (Table 3). In the ROC curve for the model, the area under the curve was 0.6727 , showing moderate accuracy of our model to predict 3-month mortality. The Hosmer-Lemeshow p value was 0.671 , indicating adequate model fit (Fig. 2).

\section{Discussion}

This study found an in-hospital mortality rate of 5.5\% and a 3-month mortality rate of $32.1 \%$ for children treated with VP shunt placement for hydrocephalus in this lowresource setting. Only younger maternal age was associated with mortality. Complications such as meningitis, shunt exposure, and shunt obstruction were not significantly associated with mortality. Of note, complications and mortality were based on family reporting and senior clerk examination; thus, follow-up complication rates and causes of mortality were sometimes difficult to assess given oftenlimited medical knowledge of the families. These results suggest that children who undergo VP shunt placement for hydrocephalus in Malawi face significant mortality rates and high rates of postoperative complications. The association of younger maternal age and increased mortality is potentially a proxy for social determinants, which may contribute as much to mortality as patient factors. The role

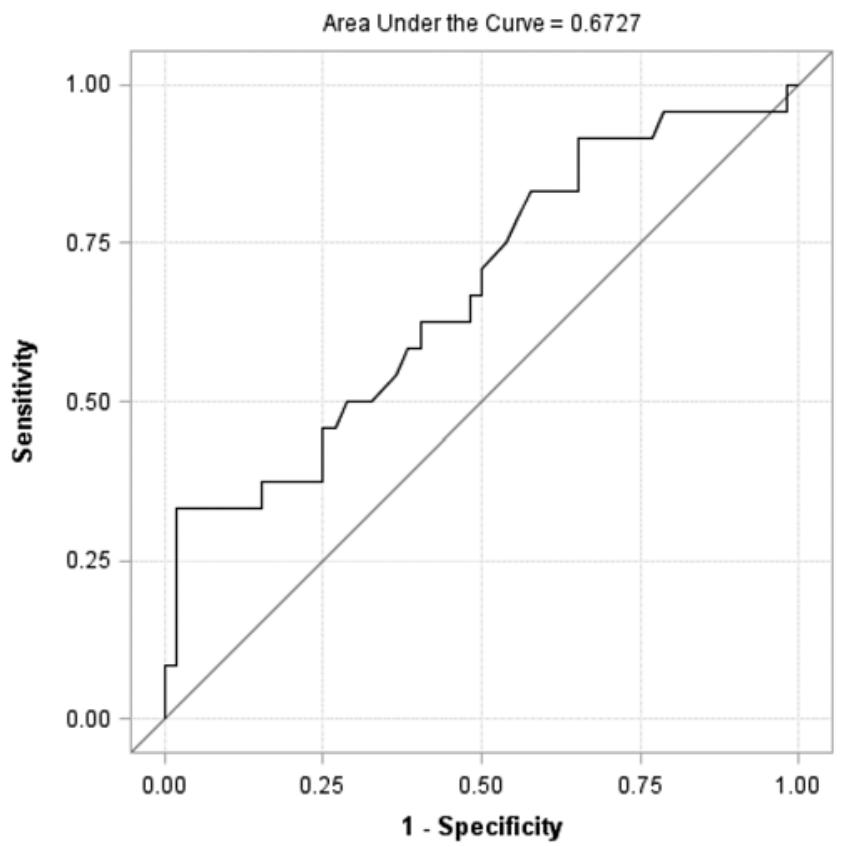

FIG. 2. ROC curve for all-cause mortality by 3 months after surgery.

that social factors play in post-shunting mortality has not been previously explored.

Few studies have evaluated morbidity and mortality related to VP shunt placement in resource-poor settings. Prior investigations similarly found substantial morbidity and mortality after shunt placement for hydrocephalus. A study of 49 children over the age of 2 years in Nigeria reported a 9.3\% shunt infection rate and an overall complication rate of $28 \% .^{8}$ Of 539 VP shunts inserted in a Kenyan hospital, complications occurred in $20 \%$, and $35 \%$ failed at 2 years. Infections occurred in $9.1 \%$ of cases, while $11 \%$ of shunts malfunctioned. The overall 9-month mortality rate reported was 7.1\%. ${ }^{10}$ Another Kenyan investigation of 117 patients over 2 years reported an obstruction rate of $54 \%$ and infection in $19.7 \%$ of patients. ${ }^{16}$ An older study reported even higher infections rates of $24.6 \% .{ }^{17}$ A study out of Ghana reported a complication rate of 34\% in 109 patients over 2 years, with infections occurring in $14.6 \%$ of patients, and an overall mortality rate of $4.6 \% .^{4}$ Our mortality rate was higher, which may be related to follow-up or to access to postoperative care. Of note, the under-5-year-old child mortality rates in Malawi for 2017 were 55 per 1000 live births compared to 46 in Kenya and 49 in Ghana. ${ }^{28}$

The reasons for such high morbidity and mortality rates in patients with shunts placed for hydrocephalus in this limited-resource setting are likely multifactorial. One possible factor is the variability of the training for the medical practitioners performing shunt surgery. At $\mathrm{KCH}, 2$ clinical officers, not a neurosurgeon, place VP shunts. Currently, there are no standard protocols for placement or training in Malawi. Successful placement is likely operator dependent, with technical factors potentially increasing infection risk. Technical factors and fragile skin both likely contribute to shunt exposures. A study in Tanzania of a neurosurgical train-forward model suggests that overall neurosurgical complications decrease significantly as the health officers' 
training progresses, which supports our concern that the complication rates after neurosurgical procedures can be highly variable depending on the expertise and experience of the proceduralist. ${ }^{7}$

No other studies have looked at social factors contributing to morbidity and mortality of shunt placement in LMICs. However, as our research suggests, these dynamics may be critically important. The reason for this association between maternal age and mortality remains unclear. Younger mothers, particularly teen mothers, may have less experience and be less apt to identify abnormal behaviors or head circumference changes, resulting in later presentation for care, as well as untimely identification of postsurgical complications after discharge. Younger mothers may also be less established socioeconomically, with access to fewer resources. While community support often plays a role in raising children in Malawi, there may be a cultural stigma associated with hydrocephalus that leads to decreased attention paid to these children. It is also possible that the physical manifestations of hydrocephalus result in neglect, particularly for the younger mothers. Indeed, in Uganda myelomeningocele patients had higher rates of neglect that may have played a significant role in mortality; this may be a significant contributing factor in our hydrocephalus patients as well. ${ }^{21}$

VP shunts are also difficult to manage postoperatively, regardless of the available resources. In HICs, shunts require frequent surveillance and often require revision. In LMICs, fewer resources are available to address complications, and children are more easily lost to follow-up, as evidenced by our inability to locate $47 \%$ of patients at 6 months despite multiple strategies to reach them in the community.

We conclude that VP shunts are inadequate as a sole surgical therapeutic strategy for infant hydrocephalus in low-resource settings. Other surgical techniques, such as endoscopic third ventriculostomy (ETV) or ETV with choroid plexus cauterization (ETV-CPC), require close followup care for early failure, but they have a very low incidence of failure beyond 6 months. ETV-CPC is more difficult to perform than VP shunt surgery and requires specialized training as well as specialized surgical equipment. While ETV-CPC may lead to smaller reductions in ventricle size when compared to VP shunts, a recent randomized controlled trial in Uganda demonstrated 12-month cognitive outcomes that were equivalent to those of VP shunts. ${ }^{12}$ Older age, hydrocephalus etiology, and more extensive CPC lead to higher success rates, allowing physicians to potentially select patients who would be ideal candidates for the procedure. ${ }^{26}$ Although longer-term data on mortality have not been studied in this cohort, the data suggest that after 3 months, ETV-CPC failure rates remain significantly lower than VP shunt rates. ${ }^{11}$ One study in Ethiopia demonstrated a 30-day mortality rate of $2.4 \%$ after ETV-CPC. ${ }^{1}$ In a Ugandan cohort, treatment was successful, at a mean follow-up of 31 months, in $82 \%$ of patients who underwent ETV-CPC, and it remained successful in $72.4 \%$ of patients at 4 years. ${ }^{24,27}$ The procedure is effective for postinfectious etiologies, which are the majority of cases in low-resource settings. ${ }^{14,25}$

\section{Study Limitations}

Limitations of the study include those inherent to any descriptive study. We cannot establish true causality and opportunity for bias exists, as this is a limited predictive model to determine associations. This population is based in one region of the country and so may not be generalizable to other regions of the country or other LMICs. Furthermore, there were missing data and loss to follow-up at 6 months of $47 \%$, despite significant efforts to locate patients, which limited our ability to determine long-term morbidity and mortality rates. We did not have enough data to evaluate all possible factors related to mortality, and reporting regarding complications was difficult given parental health literacy, which may lead to information bias. The dependence on family reporting to attribute etiology of hydrocephalus and cause of mortality makes reliability uncertain. We do not know what the prevalence of hydrocephalus in the community is, as some children may not present at all for intervention, leading to selection bias.

\section{Conclusions}

This study revealed a high 3-month mortality rate among children with hydrocephalus undergoing VP shunt placements in Malawi. Young maternal age was surprisingly a significant predictor of mortality. The impact of maternal age suggests that social determinants may affect mortality as much as patient factors in this limited-resource setting, and additional work to identify specific social factors related to mortality could guide future interventions. Further study is needed to determine if VP shunt placement can be optimized and mortality lowered in this resource-poor setting. Consideration of alternative therapies must also be contemplated given these high rates of mortality.

\section{References}

1. Biluts H, Admasu AK: Outcomes of endoscopic third ventriculostomy in pediatric patients at Zewditu Memorial Hospital, Ethiopia. World Neurosurg 92:360-365, 2016

2. Boivin MJ, Kakooza AM, Warf BC, Davidson LL, Grigorenko EL: Reducing neurodevelopmental disorders and disability through research and interventions. Nature 527:S155S160, 2015

3. Centers for Disease Control and Prevention: CDC's Developmental Milestones. CDC, U.S. Department of Health \& Human Services (https://www.cdc.gov/ncbddd/actearly/ milestones/index.html) [Accessed March 18, 2019]

4. Dakurah TK, Adams F, Iddrissu M, Wepeba GK, Akoto H, Bankah P, et al: Management of hydrocephalus with ventriculoperitoneal shunts: review of 109 cases of children. World Neurosurg 96:129-135, 2016

5. Dewan MC, Rattani A, Mekary R, Glancz LJ, Yunusa I, Baticulon RE, et al: Global hydrocephalus epidemiology and incidence: systematic review and meta-analysis. J Neurosurg 130:1065-1079, 2019

6. Drake JM, Kestle JR, Milner R, Cinalli G, Boop F, Piatt J Jr, et al: Randomized trial of cerebrospinal fluid shunt valve design in pediatric hydrocephalus. Neurosurgery 43:294-305, 1998

7. Ellegala DB, Simpson L, Mayegga E, Nuwas E, Samo H, Naman N, et al: Neurosurgical capacity building in the developing world through focused training. J Neurosurg 121:15261532, 2014

8. Emejulu JK, Ugwu JO: Combating complications following 
ventriculoperitoneal shunting in a new centre. Pediatr Neurosurg 45:446-450, 2009

9. Flannery AM, Mitchell L: Pediatric hydrocephalus: systematic literature review and evidence-based guidelines. Part 1: Introduction and methodology. J Neurosurg Pediatr 14 (Suppl 1):3-7, 2014

10. Gathura E, Poenaru D, Bransford R, Albright AL: Outcomes of ventriculoperitoneal shunt insertion in Sub-Saharan Africa. J Neurosurg Pediatr 6:329-335, 2010

11. Kulkarni AV, Drake JM, Kestle JR, Mallucci CL, Sgouros S, Constantini S: Endoscopic third ventriculostomy vs cerebrospinal fluid shunt in the treatment of hydrocephalus in children: a propensity score-adjusted analysis. Neurosurgery 67:588-593, 2010

12. Kulkarni AV, Schiff SJ, Mbabazi-Kabachelor E, Mugamba J, Ssenyonga P, Donnelly R, et al: Endoscopic treatment versus shunting for infant hydrocephalus in Uganda. N Engl J Med 377:2456-2464, 2017

13. Laurence KM, Coates S: The natural history of hydrocephalus. Detailed analysis of 182 unoperated cases. Arch Dis Child 37:345-362, 1962

14. Mugamba J, Stagno V: Indication for endoscopic third ventriculostomy. World Neurosurg 79 (2 Suppl):20.e19-20.e23, 2013

15. Muir RT, Wang S, Warf BC: Global surgery for pediatric hydrocephalus in the developing world: a review of the history, challenges, and future directions. Neurosurg Focus 41(5):E11, 2016

16. Mwachaka PM, Obonyo NG, Mutiso BK, Ranketi S, Mwang'ombe N: Ventriculoperitoneal shunt complications: a three-year retrospective study in a Kenyan national teaching and referral hospital. Pediatr Neurosurg 46:1-5, 2010

17. Mwang'ombe NJ, Omulo T: Ventriculoperitoneal shunt surgery and shunt infections in children with non-tumour hydrocephalus at the Kenyatta National Hospital, Nairobi. East Afr Med J 77:386-390, 2000

18. Paulsen AH, Lundar T, Lindegaard KF: Pediatric hydrocephalus: 40-year outcomes in 128 hydrocephalic patients treated with shunts during childhood. Assessment of surgical outcome, work participation, and health-related quality of life. J Neurosurg Pediatr 16:633-641, 2015

19. Salvador SF, Henriques JC, Munguambe M, Vaz RM, Barros HP: Hydrocephalus in children less than 1 year of age in northern Mozambique. Surg Neurol Int 5:175, 2014

20. Simon TD, Hall M, Riva-Cambrin J, Albert JE, Jeffries HE, Lafleur B, et al: Infection rates following initial cerebrospinal fluid shunt placement across pediatric hospitals in the United States. Clinical article. J Neurosurg Pediatr 4:156-165, 2009

21. Sims-Williams HJ, Sims-Williams HP, Kabachelor EM, Fotheringham J, Warf BC: Ten-year survival of Ugandan infants after myelomeningocele closure. J Neurosurg Pediatr 19:70-76, 2017
22. Townsend CM, Beauchamp RD, Evers BM, Mattox KL (eds): Sabiston Textbook of Surgery, ed 20. Philadelphia: Elsevier Saunders, 2017

23. Tunkel AR, Hasbun R, Bhimraj A, Byers K, Kaplan SL, Michael Scheld W, et al: 2017 Infectious Diseases Society of America's clinical practice guidelines for healthcare-associated ventriculitis and meningitis. Clin Infect Dis 64:e34e65, 2017

24. Warf BC: Congenital idiopathic hydrocephalus of infancy: the results of treatment by endoscopic third ventriculostomy with or without choroid plexus cauterization and suggestions for how it works. Childs Nerv Syst 29:935-940, 2013

25. Warf BC: Hydrocephalus in Uganda: the predominance of infectious origin and primary management with endoscopic third ventriculostomy. J Neurosurg 102 (1 Suppl):1-15, 2005

26. Warf BC, Mugamba J, Kulkarni AV: Endoscopic third ventriculostomy in the treatment of childhood hydrocephalus in Uganda: report of a scoring system that predicts success. J Neurosurg Pediatr 5:143-148, 2010

27. Warf BC, Tracy S, Mugamba J: Long-term outcome for endoscopic third ventriculostomy alone or in combination with choroid plexus cauterization for congenital aqueductal stenosis in African infants. J Neurosurg Pediatr 10:108-111, 2012

28. The World Bank: Mortality rate, under-5 (per 1,000 live births). The World Bank. The World Bank Group (https://data.worldbank.org/indicator/SH.DYN. MORT?locations=MW-GH-KE) [Accessed March 18, 2019]

\section{Disclosures}

The authors report no conflict of interest concerning the materials or methods used in this study or the findings specified in this paper.

\section{Author Contributions}

Conception and design: Grudziak, Charles. Acquisition of data: Grudziak, Msiska. Analysis and interpretation of data: Reid, Rodriguez-Ormaza, Maine, Quinsey, Charles. Drafting the article: Reid, Grudziak. Critically revising the article: Reid, Grudziak, Rodriguez-Ormaza, Maine, Quinsey, Charles. Reviewed submitted version of manuscript: all authors. Approved the final version of the manuscript on behalf of all authors: Reid. Statistical analysis: Rodriguez-Ormaza. Administrative/technical/material support: Reid, Msiska. Study supervision: Charles.

\section{Correspondence}

Trista Reid: UNC School of Medicine, University of North Carolina, Chapel Hill, NC. trista_reid@med.unc.edu. 